\title{
Disruption Mitigation by Massive Gas Injection in JET
}

M. Lehnen ${ }^{1 *}$, A. Alonso ${ }^{2}$, G. Arnoux ${ }^{3}$, N. Baumgarten ${ }^{1}$, S.A. Bozhenkov ${ }^{4}$, S. Brezinsek ${ }^{1}$, M. Brix ${ }^{3}$, T. Eich ${ }^{4}$, S.N. Gerasimov ${ }^{3}$, A. Huber ${ }^{1}$, S. Jachmich ${ }^{5}$, U. Kruezi ${ }^{1}$, P.D. Morgan ${ }^{3}$, V.V. Plyusnin ${ }^{6}$, C. Reux ${ }^{7}$, V. Riccardo ${ }^{3}$, G. Sergienko ${ }^{1}$, M.F. Stamp ${ }^{3}$, A. Thornton ${ }^{8}$, M. Koltunov ${ }^{1}$, M. Tokar ${ }^{1}$, B. Bazylev ${ }^{9}$, I. Landman ${ }^{9}$, S. Pestchanyi ${ }^{9}$ and JET EFDA contributors ${ }^{\dagger}$

JET-EFDA, Culham Science Centre, OX14 3DB, Abingdon, UK

${ }^{1}$ Institute for Energy Research - Plasma Physics,Forschungszentrum Jülich, Association EURATOM-FZJ, Trilateral Euregio Cluster, 52425 Jülich, Germany; ${ }^{2}$ Laboratorio Nacional de Fusion, Asociacion EURATOM-CIEMAT, Madrid, Spain; ${ }^{3}$ Euratom/CCFE Association, Culham Science Centre, Abingdon, Oxon, OX14 3DB, UK; ${ }^{4}$ Max-Planck-Institut für Plasmaphysik, EURATOM-Assoziation, D-85748 Garching, Germany; ${ }^{5}$ Laboratoire de Physique des Plasmas-Laboratorium voor Plasmafysica, Association EURATOM-Belgian State, ERM/KMS, B-1000 Brussels, Belgium; ${ }^{6}$ Instituto de Plasmas e Fusão Nuclear/IST, Associacao EURATOM-IST, Av. Rovisco Pais, 1049-001 Lisbon, Portugal; ${ }^{7}$ CEA, IRFM, F-13108 Saint-Paul-lez-Durance, France; ${ }^{8}$ Department of Physics, University of York, Heslington, York, YO10 5DD, UK ${ }^{9}$ Karlsruhe Institute of Technology, IHM, D-76021 Karlsruhe, Germany

\begin{abstract}
Disruption mitigation is mandatory for ITER in order to reduce forces and to mitigate heat loads during the thermal quench (TQ) and from runaway electrons. A fast disruption mitigation valve (DMV) has been installed at JET to study mitigation by massive gas injection (MGI). Different gas species and amounts have been investigated with respect to timescales and mitigation efficiency. Halo currents as well as sideways forces during VDE are successfully reduced by MGI. About 50\% of the thermal energy is dissipated by radiation during a MGI disruption. The radiation is homogeneously distributed poloidally, with a peaking factor below 1.5 during thermal and current quench. Significant poloidal and toroidal peaking of up to 2.5 is observed before the thermal quench. Runaway generation is successfully avoided by the injection of mixtures of $\mathrm{Ar}$ or $\mathrm{Ne}$ with $\mathrm{D}_{2}$. In contrast, injection of pure Ar leads to runaway generation even at low toroidal magnetic fields. Although, runaways can be safely avoided by MGI in JET disruptions, the density reached is a factor 50 below the critical density for avalanche suppression, which will be essential in ITER.
\end{abstract}

\section{Introduction}

The mitigation of thermal and mechanical loads during disruptions is an urgent task to be solved for ITER to ensure the integrity of plasma-facing components (PFC). However, extreme loads are already an issue for present day machines like JET, with its new ITERlike wall, having the material used in ITER for DT operation [1]. Reduction of such loads to tolerable values is needed, consequently the generation of high-energy electrons (socalled runaway electrons - RE) is of special concern. RE in JET can carry currents of up to $50 \%$ of plasma current before the disruption, leading to a fast and localised deposition of several MJ on main chamber PFC $[2,3]$.

Disruption mitigation has to fulfill three aims: mitigation of forces from halo and eddy currents, mitigation of convective/conductive heat loads during the thermal quench, mitigation of heat loads from runaway electrons. ITER needs a reduction of the forces by a factor $2-3$ and a reduction of the thermal loads on Be and $\mathrm{W}$ components by at least a factor 10 to ensure integrity and lifetime of PFC [4].

A fast valve (Disruption Mitigation Valve - DMV) has been installed at JET to study disruption mitigation by massive gas injection $[5,6,7,8]$. The valve is positioned on top

\footnotetext{
*email: m.lehnen@fz-juelich.de

${ }^{\dagger}$ See the Appendix of F. Romanelli et al., paper OV/1-3, this conference
} 


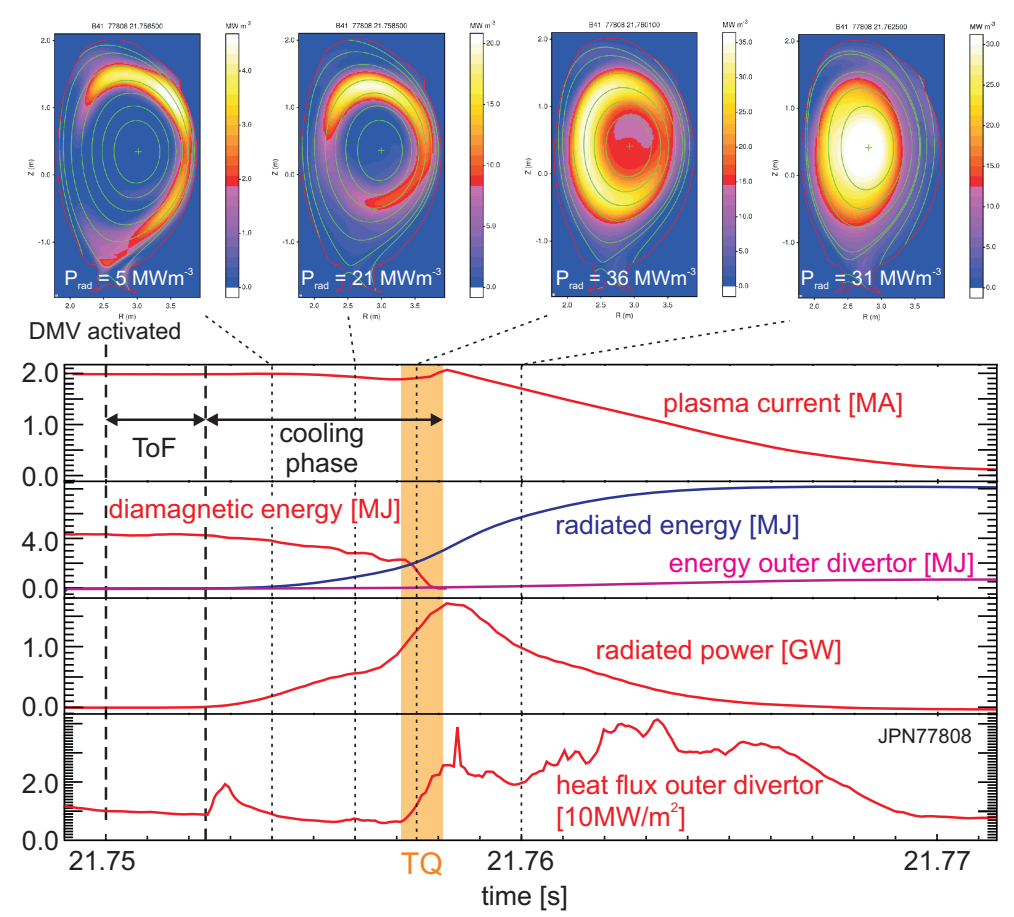

Figure 1: Disruption sequence. Ar $/ \mathrm{D}_{2}$ injection, $p_{D M V}=3.6 \mathrm{MPa}$.

of the machine and the gas is guided by a $4 \mathrm{~m}$ long tube to the plasma. Gas species investigated were helium, neon, argon and mixtures of these with $90 \%$ of deuterium as well as pure deuterium. A maximum of $2.5 \times 10^{23}$ particles can be injected, corresponding to about 100 times the electron content in the plasma.

Figure 1 shows the sequence of a typical JET disruption triggered by injection of about $2 \times 10^{23}$ particles of the $\mathrm{Ar} / \mathrm{D}_{2}$ mixture into a NBI heated plasma. After the activation of the DMV, the gas flows through the tube and arrives after a delay of about $2 \mathrm{~ms}$ at the plasma edge. At that time the cooling of the plasma edge starts and part of the thermal energy is dissipated by radiation. Eventually, the thermal quench is triggered when the cooling front arrives at a critical flux surface and the remaining thermal energy is released within less than $1 \mathrm{~ms}$. The thermal quench is followed by the decay of the plasma current caused by the high resistivity of the remaining low temperature plasma.

\section{Pre-thermal-quench phase}

The pre-thermal-quench phase includes the time-of-flight (TOF) of the gas from the valve to the plasma edge and the duration of the edge cooling process prior to the initiation of the thermal quench (TQ). The TOF depends on the sound speed $c_{0}$ of the injected species and thus mainly on the mass. From the theory of adiabatic expansion into vacuum, the gas front arrives at the plasma edge after a time: $\Delta t=L / 3 c_{0}$, where $L=4.5 \mathrm{~m}$ and $c_{0}=\sqrt{\gamma R T / M}$. The minimum TOF, as found in the experiment by taking the decay of the electron temperature in the outermost channel of the ECE diagnostic as indicator for the gas arrival [5], gives $\Delta t \approx L / 2.5 c_{0}$. The time of flight varies from about $6 \mathrm{~ms}$ for argon to $2 \mathrm{~ms}$ for a mixture of $10 \%$ neon and $90 \%$ deuterium.

Figure 2a shows the cooling duration for various species and pressure. This time is defined as the delay between DMV activation and the start of the current quench (CQ) as indicated by the positive peak in the plasma current and includes therefore also the duration of the thermal quench. The TOF has been subtracted. The cooling duration decreases not only with the number of injected particles, but also with the safety factor $q_{95}$, indicating that the thermal quench is initiated when the cooling front reaches a critical 


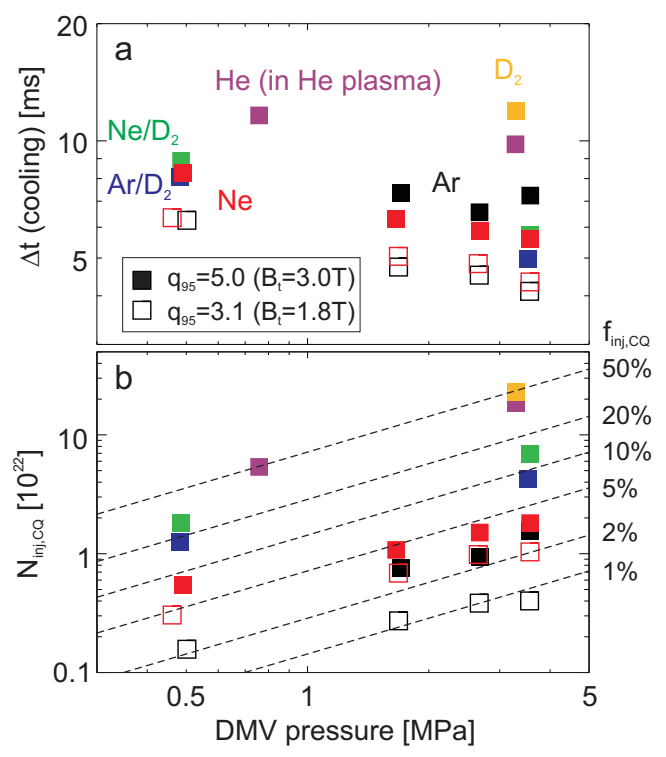

Figure 2: Duration of the cooling phase (a) and calculated number of injected particles before the current quench (b).

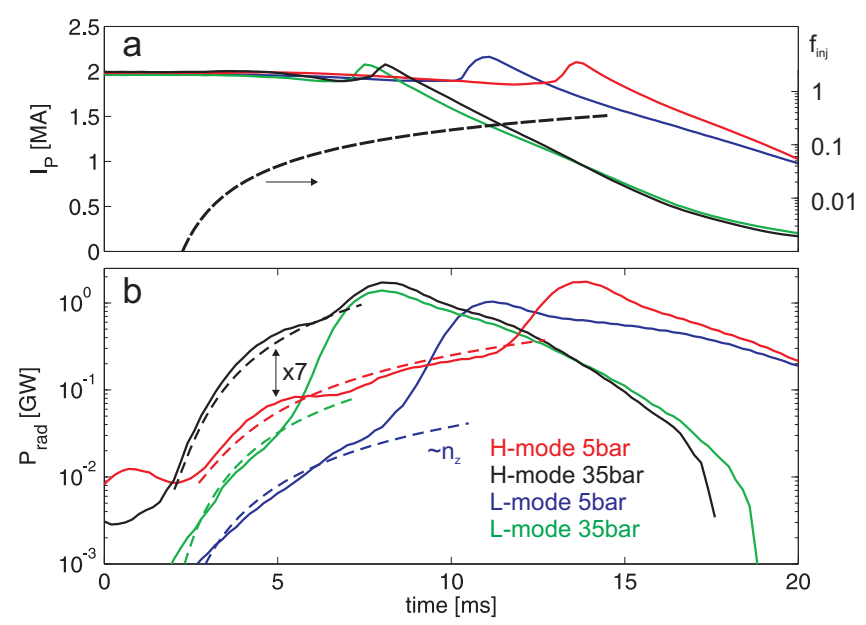

Figure 3: Plasma current (a) and radiation (b) during MGI for $\mathrm{Ar} / \mathrm{D}_{2}$ injection. The dashed line in (a) gives the fraction of injected gas. The $D M V$ is activated at $t=0$.

flux surface (presumably $q=2$ ).

The duration of the cooling phase is important for the efficiency of the gas injection. The greater the quantity of injected gas, the shorter the pre-TQ phase and, therefore, less time is available for the gas injection. The assimilation of gas injected after the thermal quench is assumed to be much less efficient and this gas can also not contribute to the mitigation of heat loads during the TQ. Figure $2 \mathrm{~b}$ gives the number of particles injected before start of the current quench $N_{i n j, C Q}$ as calculated from adiabatic expansion into vacuum. This approach has been validated with lab measurements using the JET set-up $[9,10,11]$. The fraction of gas injected before the current quench $f_{i n j, C Q}$ decreases with increasing pressure, because of the shorter cooling phase. However, an increase in the absolute number of particles is still achieved.

Figure 3 shows the temporal evolution of the plasma current and the radiated power during $\mathrm{Ar} / \mathrm{D}_{2}$ injection into L- and $\mathrm{H}$-mode discharges, for the minimum and maximum pressure in the DMV. Although the waveform of the gas injection is identical for all four disruptions and, thus, is the amount of injected impurities, the radiated power $P_{\text {rad }}$ is in the first phase of the cooling process more than one order of magnitude lower for the L-mode disruptions. This could be partly attributed to the low initial electron density, which is about 3 times higher in the H-mode cases. In this first phase, $P_{\text {rad }}$ increases only linearly with the injected amount of gas as indicated by the dashed lines in $3 \mathrm{~b}$, which represent the fraction of injected gas $f_{i n j}$ times a factor to fit the measured $P_{\text {rad }}$. In the H-mode disruptions, $P_{\text {rad }}$ increases proportional to the valve pressure by a factor 7 , in L-mode the increase is about a factor 4 . In the later phase of the cooling process a strongly non-linear increase of $P_{r a d}$ is observed about $1-2 \mathrm{~ms}$ before the current spike. The large difference in $P_{\text {rad }}$ results in a similar cooling duration for L- and H-mode with strong MGI $\left(p_{D M V}=3.5 \mathrm{MPa}\right)$, despite the significant difference in thermal energy: $E_{t h} \approx 4.5 \mathrm{MJ}$ (H-mode) / 1.0 MJ (L-mode). 


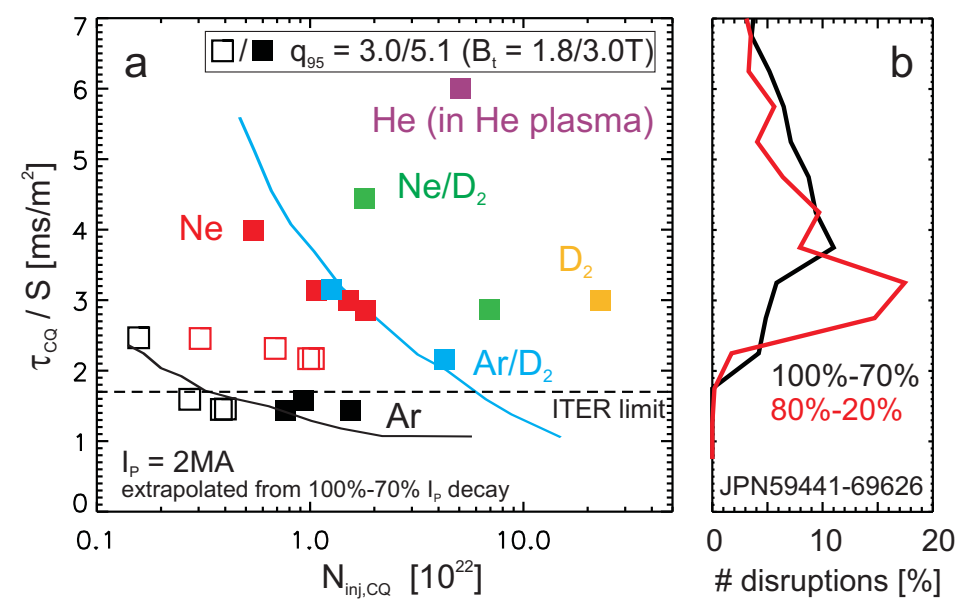

Figure 4: Linear current decay time for MGI (a) and reference database (b).

\section{Mitigation of forces}

Massive gas injection aims at a fast current decay in order to mitigate forces from halo currents. Figure 4 shows the linear current decay time extrapolated from the drop in current from $100 \%$ to $70 \%$ of the pre-TQ current and normalised to the plasma cross section. This definition has been chosen in order to avoid any influence from runaway current plateaux. In [12] it has been shown that the extrapolation range can have strong impact on the estimated decay time. Therefore, we compare in figure $4 \mathrm{~b}$ with non-MGI references for both definitions, the 'standard' $80 \%$ to $20 \%$ decay and our definition. MGI causes a faster current decay in comparison to most reference disruptions. From the tendency of the standard definition to result in shorter $\tau_{C Q}$ it becomes obvious that the CQ in many 'natural' disruptions starts with a slow current decay (most likely because of a weak impurity influx), which accelerates in the later phase of the CQ. With MGI, the current decay is fast from the very beginning of the CQ, which is essential for the mitigation of vertical displacement events (VDE). In order to keep forces from eddy currents tolerable, the current decay time has to stay above the lower bound of $\tau_{C Q} / S=1.7 \mathrm{~ms} / \mathrm{m}^{2}$ for ITER, which has been defined for the $80 \%$ to $20 \%$ decay. This limit was reached with pure Ar MGI in JET, however, the different definition of $\tau_{C Q}$ chosen here can lead to a lower $\tau_{C Q}$. For $\mathrm{D}_{2}$ mixtures, where runaway generation is avoided, the difference between the two definitions amounts to about $20 \%$. A saturation of $\tau_{C Q} / S$ at larger numbers of injected particles is not obvious from the present database.

Halo currents can generate strong forces on the vessel and on inner wall structures. Especially, the product of halo current fraction $I_{\text {halo }} / I_{P}$ and toroidal peaking factor TPF has to be limited to ensure the integrity of ITER wall components. These halo currents are reduced by MGI, if the thermal quench is initiated before the vertical position has moved significantly in a VDE. Figure 5 shows the halo current fraction multiplied by the toroidal peaking factor as function of the delay between thermal quench and a vertical displacement of $10 \mathrm{~cm}$ for a fast VDE $\left(\tau_{\text {growth }} \approx 5 \mathrm{~ms}\right)$. Essential for a successful mitigation of halo currents is a fast reaction time. This time depends on the time of flight in the delivery tube and the duration of the cooling phase until thermal quench as described in section 2 . The minimum reaction time of $6 \mathrm{~ms}$, achieved with the $\mathrm{Ar} / \mathrm{D}_{2}$ mixture, allows reduction of the halo currents by $60 \%$. Beside halo currents, sideways forces caused by toroidal asymmetries in the vertical current moment $\delta M_{z}=\delta\left(I_{p} z_{p}\right)$ during the current quench are of concern for ITER $[13,14]$. The normalised sideways impulse $\int_{C Q} \delta M_{z} d t / 2 I_{p} a_{p}$ (integrated over the current quench) is reduced by more than an order of magnitude (figure $5)$. 


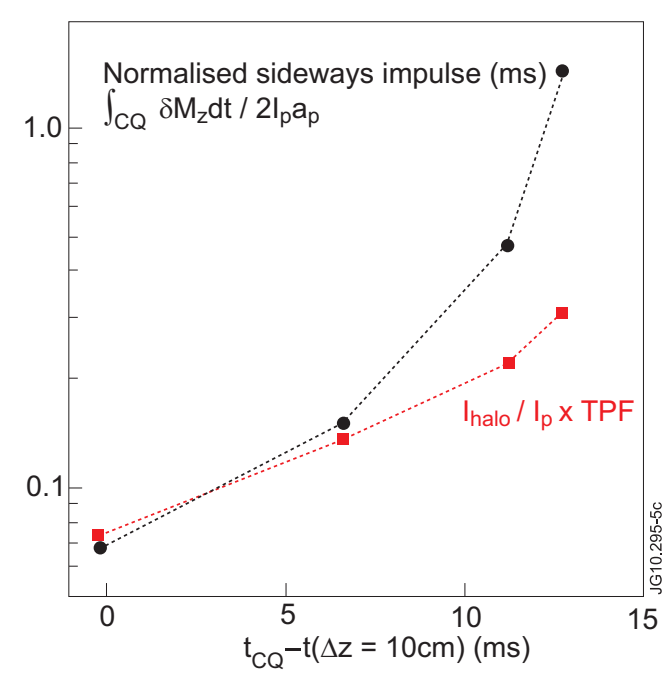

Figure 5: Reduction of halo currents and sideways forces.

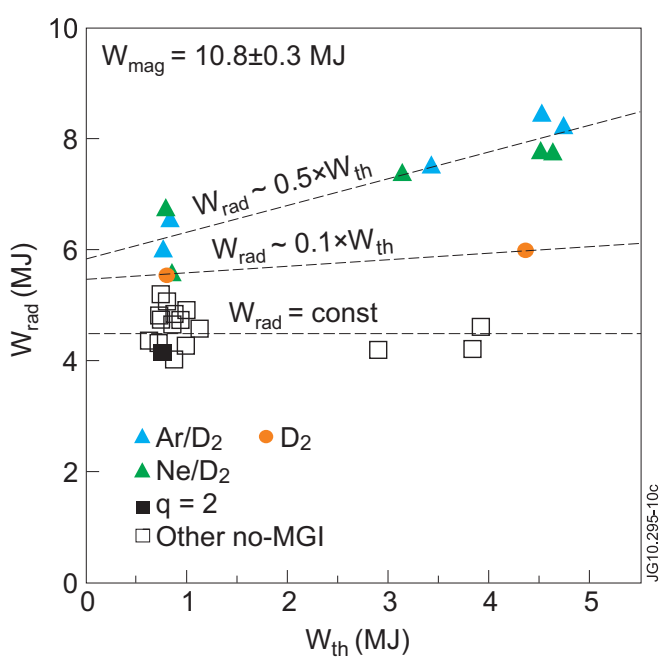

Figure 6: Energy radiated during the disruption as function of initial stored thermal energy.

\section{Mitigation of heat loads}

Heat loads during the thermal quench can be reduced by enhancing the radiation with MGI. This is in contrast to non-MGI disruptions, where the impurities are released during the thermal quench by the high heat fluxes to the divertor or other PFC. The time traces in figure 1 show that with $\mathrm{Ar} / \mathrm{D}_{2}$ injection into $\mathrm{H}$-mode plasma up to $50 \%$ of the thermal energy is lost predominantly by radiation before the TQ. About $40 \%$ of the remaining energy is radiated during the TQ. Thus, $30 \%$ of the initial energy is lost by convection during the TQ to the PFC and only about $5 \%$ of this $30 \%$ is found in the (outer) divertor, the rest is distributed to main chamber PFC [3].

Because the above estimates are influenced by the uncertainties in separating the different disruption phases in conjunction with the steep increase in $P_{\text {rad }}$ and limited time resolution of the bolometer, another method is used to determine the fraction of radiated energy during MGI. The energy balance is done by comparing the radiated energy during the whole disruption with the thermal and magnetic energy stored in the plasma before the disruption. The radiated energy is

$$
W_{\text {rad }}=f_{\text {mag }} \times\left(W_{\text {mag }}-W_{\text {mag }}^{\text {structure }}-W_{m a g}^{R E}\right)+f_{t h} \times W_{t h},
$$

with $f_{\text {mag }} \approx 1$; it was shown for JET that almost $100 \%$ of the ohmic power during the current quench is dissipated by radiation [15]. In the following, we analyse disruptions with $\mathrm{Ne} / \mathrm{D}_{2}$ and $\mathrm{Ar} / \mathrm{D}_{2}$ injection, which show no generation of runaways $\left(W_{\text {mag }}^{R E}=0\right)$. In figure 6 the radiated energy is shown as function of the thermal energy. The plasma current is $2 \mathrm{MA}$, the magnetic energy $W_{\text {mag }}=10.8 \pm 0.3 \mathrm{MJ}$, accordingly. With the assumption that the dissipation of magnetic energy in the structure $W_{\text {mag }}^{\text {structure }}$ is constant for these disruptions, we find that about $50 \%$ of the stored thermal energy is dissipated by radiation, which is within the error bars consistent with the estimate given above. For comparison, disruptions with slow gas injection $\left(10^{22}\right.$ atoms/s), a $q=2$ disruption, caused by deliberate ramp-down of the toroidal magnetic field, and natural disruptions from a broader JET database with the same magnetic energy are shown. The reference disruptions without gas injection show no dependence on $W_{t h}$ and a higher $W_{\text {mag }}^{\text {structure }}$ as seen from the extrapolated offset in $W_{\text {rad }}$ at $W_{t h}=0$.

Beside the heat loads caused by convection/conduction during the thermal quench, radiation could also lead to heat fluxes close to the PFC melting limits in ITER. During 


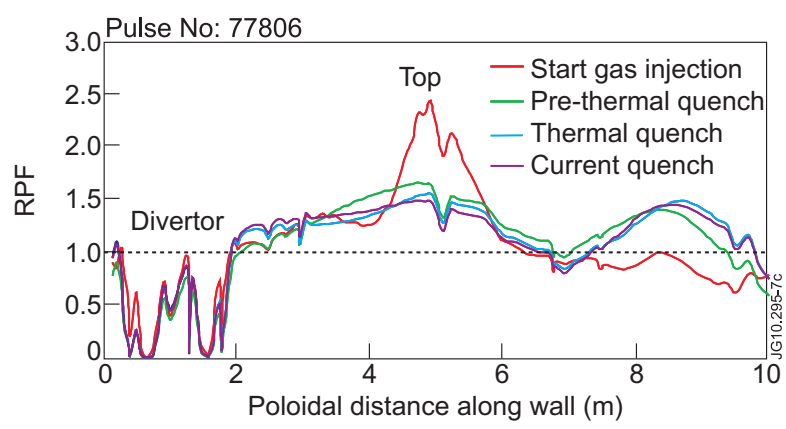

Figure 7: Poloidal radiation peaking during $\mathrm{Ar} / \mathrm{D}_{2}$ injection into H-mode plasma [16].

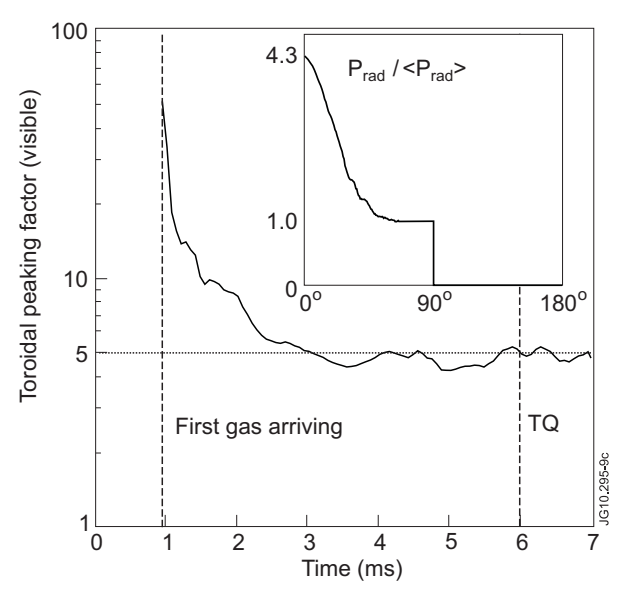

Figure 8: Toroidal peaking from visible emission (Pulse No 76321).

natural disruptions in JET, strong poloidal peaking of the heat fluxes by radiation have been reported, with peaking factor of up to 3.5 during VDE [16]. With MGI, the radiation is very localised when the first gas arrives, but extents poloidally and toroidally in the later phase and generates a radiating mantle around the remaining core plasma just before the thermal quench (see figure 1). In the current quench, most of the radiation is found in the plasma center. The poloidal peaking factor is below 1.7 for most of the disruption phases (figure 7). However, beryllium melting by local radiation at the injection port is of concern for ITER and has to be addressed to determine the number of injection ports. Indeed, a strong poloidal peaking of up to 2.5 is found in the pre-TQ phase in JET MGI experiments (figure 7). In order to estimate the toroidal peaking, visible radiation recorded by a fast camera viewing the injection section has been analysed. We assume that the total radiation consists only of visible emission, neglecting therefore the emission from ions with higher degree of ionisation, which have a broader distribution around the injection point. The bolometry, $90^{\circ}$ toroidally separated from the injection port, measures a radiated energy during the pre-TQ phase equal to about $80-100 \%$ of the thermal energy loss. Radiation for $\phi>90^{\circ}$ is assumed to be zero. From these assumptions, we get an upper bound for the peaking factor from the toroidal profile of the visible radiation as shown in figure 8. The peaking is quite strong during the initial phase, but reduces to about 5.0 for the later phase with higher $P_{r a d}$. The present estimate for ITER for the pre-TQ phase is a loss of thermal energy of $W_{\text {pre-TQ }}=180 \mathrm{MJ}$ by radiation within $7 \mathrm{~ms}$ [4]. With toroidal and poloidal peaking factors of 5 and 2.5, respectively, we find a heat load parameter of $40 \mathrm{MJs}^{-0.5} \mathrm{~m}^{-2}$ for a single injection point. The Be melting limit of about $15 \mathrm{MJs}^{-0.5} \mathrm{~m}^{-2}$ suggests the use of at least 4 injection ports in ITER.

\section{Generation and mitigation of runaway electrons}

Runaway generation is observed with injection of pure neon and argon. Argon injection leads for all possible gas amounts to a current plateau with up to $1 \mathrm{MA}$ of runaway current for the 2 MA pulses discussed in this paper. Traces of runaways indicated by neutron radiation can be found in Ar MGI even at magnetic fields down to $1.2 \mathrm{~T}$. Runaway generation during Neon injection is much weaker and occurs in most cases in the tail of the current quench phase. No runaway generation is observed during injection of the deuterium mixtures.

In present-day tokamaks the primary runaway electrons during disruptions are generated by the Dreicer mechanism. In ITER other sources of runaway electrons will exist. The primary runaways are then multiplied by the avalanche process. Suppression of the Dreicer 


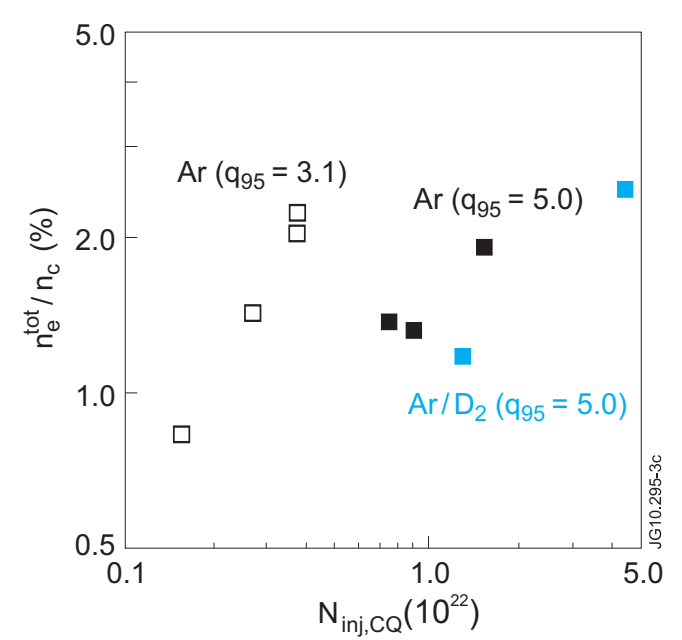

Figure 9: Total electron density normalised to the critical density for unconditional suppression of runaway electrons, which has been calculated from the maximum electric field during the $C Q$.

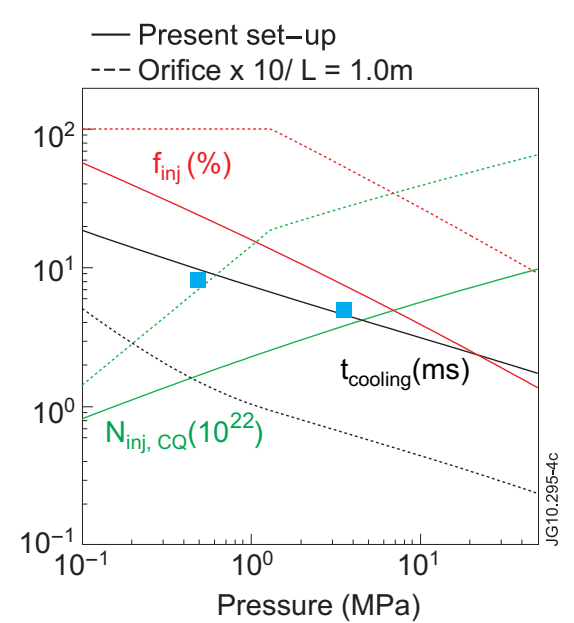

Figure 10: Extrapolation of gas injection towards runaway suppression. Blue squares mark the measured $\Delta t_{\text {cooling }}$ for $\mathrm{Ar} / \mathrm{D}_{2}$.

mechanism happens at densities of the order of $10^{20} \mathrm{~m}^{-3}$ in JET, whereas the suppression of the avalanche requires total electron densities (free and bound electrons) above $n_{c}=10^{21} \mathrm{~m}^{-3} \times E / \mathrm{Vm}^{-1}$. Such densities can only be achieved with a sufficiently high assimilation of the injected particles in the plasma. The impurity density in the current quench plasma can be estimated from the current decay time by using a simple model for the current decay, which includes plasma and vessel current as well as the power balance $P_{\text {rad }}=P_{O H}$ [17]. This model can be applied, if the radiation is predominantly caused by the injected species, which is expected for argon. The linear current quench times have been calculated as function of the number of impurities in the plasma. These values were fitted to the measured $\tau_{C Q}$ (blue and black line in figure 4), resulting in an assimilation efficiency $N_{\text {plasma }} / N_{i n j, C Q}$ of $50 \pm 10 \%\left(A r / D_{2}\right)$ and $65 \pm 35 \%$ (Ar). With Ar injection we see a tendency towards higher assimilation efficiencies with decreasing $q_{95}$. The same trend can be seen for Ne, where a shorter $\tau_{C Q}$ is achieved for $q_{95}=3.1$.

The fraction of critical density achieved with $\mathrm{Ar} / \mathrm{D}_{2}$ and pure $\mathrm{Ar}$ injection is given in figure 9. With the present set-up, MGI at JET reaches only $2 \%$ of the critical density. Extrapolation to a more advanced injection system can be done by assuming that the thermal quench is initiated after a certain energy $W_{p r e-T Q}$ has been dissipated by radiation:

$$
W_{\text {pre-TQ }}=\int_{0}^{\Delta t_{\text {pre-TQ }}} P_{\text {rad }} \sim \int_{0}^{\Delta t_{\text {pre-TQ }}} N_{Z},
$$

the latter proportionality being justified by the observations discussed in section 2. From this, we estimate the cooling duration as function of the pressure in the DMV $\left(N_{Z} \sim\right.$ $\left.p \times f_{i n j}\right)$, which is given in figure 10. The decrease of $\Delta t_{p r e-T Q}$ with increasing pressure fits well to the experimental data points (blue squares, taken from figure 2), giving confidence to this approach. These calculations show that a further increase of the pressure will indeed increase $N_{i n j, C Q}$, but is technically limited. Further increase can only be achieved by enlarging the valve orifice and/or a shortening of the distance between valve and plasma. An example with orifice diameter of $10 \mathrm{~cm}$ and only $1 \mathrm{~m}$ distance to plasma is given in figure 10, showing that with this already demanding set-up an increase of $N_{i n j, C Q}$ by a factor 10 could be feasible, still not enough to reach the critical density. However, this simplified estimate is done on the basis that the relation $P_{r a d} \sim N_{Z}$ can be extrapolated to these amounts of gas. This might be a pessimistic assumption, because plasma parameters 
like the finite initial electron density might cause a saturation in the radiated power. It might as well be too optimistic, because it assumes that the assimilation efficiency does not degrade with the number of injected particles. If this relation holds, it would not be feasible to reach the critical density in JET due to the required valve dimensions (extremely large orifice to be opened on a sub-millisecond timescale). Experiments with higher $N_{i n j, C Q}$ are urgently needed to draw a conclusion for ITER.

\section{Summary and Conclusions}

The experiments with massive gas injection at JET have shown that this concept is able to significantly reduce the loads during disruptions. Halo currents are reduced by up to a factor of 4, sideways forces even by more than a factor of 10 during VDE. The heat loads during the thermal quench can be reduced by the enhanced radiation, which dissipates about $50 \%$ of the stored thermal energy. The radiation peaking is reduced to an acceptable value during most part of the disruption. A strong poloidal and toroidal peaking is observed for the pre-TQ phase. Conservative extrapolation to ITER suggests that 4 injection ports are needed to stay below the Be melting limit. Runaway generation is successfully avoided by injecting mixtures of $\mathrm{Ne}$ or $\mathrm{Ar}$ with $90 \%$ deuterium. However, only $2 \%$ of the critical density for the suppression of the runaway avalanche is achieved. Extrapolation from the measured duration of the pre-TQ phase shows that a large valve outlet and a short distance to the plasma are essential to approach these amounts of impurities.

This work was supported by EURATOM and carried out within the framework of the European Fusion Development Agreement. The views and opinions expressed herein do not necessarily reflect those of the European Commission.

\section{References}

[1] G.F. Matthews et al., Phys. Scr. T138 (2009) 014030

[2] M. Lehnen et al., J. Nucl. Mater. 390-391 (2009) 740.

[3] G. Arnoux et al., 19th PSI conference 2010.

[4] M. Sugihara, IEA Workshop on Key ITER Disruption Issues, Culham, October 2009.

[5] U. Kruezi et al., 36th EPS Conference on Plasma Phys. Sofia, June 29 - July 3, ECA 33E, P-2.153 (2009).

[6] M. Lehnen et al., 36th EPS Conference on Plasma Phys. Sofia, June 29 - July 3, ECA 33E, O-2.001 (2009).

[7] S.A. Bozhenkov et al, JET Experiments on Massive Gas Injection, 51st APS Meeting, Atlanta, 2009

[8] U. Kruezi et al., 19th PSI conference, San Diego (2010).

[9] S.A. Bozhenkov et al., 36th EPS Conference on Plasma Phys. Sofia, June 29 - July 3, ECA 33E, P-1.180 (2009)

[10] K.H. Finken et al., submitted to Nucl. Fusion. (2010).

[11] S.A. Bozhenkov et al., to be published (2010).

[12] V. Riccardo et al., Plasma Phys. Control. Fusion 47 (2005) 117.

[13] S.N. Gerasimov et al., Scaling JET disruption sideways forces to ITER, EPS 2010 Dublin, P4.121.

[14] T.C. Hender et al., this conference, EXS/10-3.

[15] J.I. Paley et al., J. Nucl. Mater. 337-339 (2005) 702.

[16] A. Huber et al., 19th PSI conference, San Diego (2010).

[17] S.A. Bozhenkov et al., Plasma Phys. Control. Fusion 50 (2008) 105007. 